PROCEEDINGS OF THE

AMERICAN MATHEMATICAL SOCIETY

Volume 140, Number 6, June 2012, Pages 2075-2089

S 0002-9939(2011)11066-9

Article electronically published on October 19, 2011

\title{
BASIC HYPERGEOMETRIC FUNCTIONS AND ORTHOGONAL LAURENT POLYNOMIALS
}

\author{
MARISA S. COSTA, EDUARDO GODOY, REGINA L. LAMBLÉM, AND A. SRI RANGA
}

(Communicated by Walter Van Assche)

\begin{abstract}
A three-complex-parameter class of orthogonal Laurent polynomials on the unit circle associated with basic hypergeometric or $q$-hypergeometric functions is considered. To be precise, we consider the orthogonality properties of the sequence of polynomials $\left\{{ }_{2} \Phi_{1}\left(q^{-n}, q^{b+1} ; q^{-c+b-n} ; q, q^{-c+d-1} z\right)\right\}_{n=0}^{\infty}$, where $0<q<1$ and the complex parameters $b, c$ and $d$ are such that $b \neq-1,-2, \ldots, c-b+1 \neq-1,-2, \ldots, \mathcal{R} e(d)>0$ and $\mathcal{R} e(c-d+2)>0$. Explicit expressions for the recurrence coefficients, moments, orthogonality and also asymptotic properties are given. By a special choice of the parameters, results regarding a class of Szegő polynomials are also derived.
\end{abstract}

\section{INTRODUCTION}

Given the double sequence $\left\{\mu_{n}\right\}_{n=-\infty}^{\infty}$ of complex numbers, let the linear functional $\mathcal{M}$ on the space of Laurent polynomials be defined by

$$
\mathcal{M}\left[z^{-n}\right]=\mu_{n}, \quad n=0, \pm 1, \pm 2, \ldots .
$$

The functional $\mathcal{M}$ can be referred to as a moment functional.

Let $D_{n}, n=0,1, \ldots$, be the associated Toeplitz determinants as given by:

$$
D_{-1}=1, \quad D_{0}=\mu_{0} \quad \text { and } \quad D_{n}=\left|\begin{array}{cccc}
\mu_{0} & \mu_{-1} & \cdots & \mu_{-n} \\
\mu_{1} & \mu_{0} & \cdots & \mu_{-n+1} \\
\vdots & \vdots & & \vdots \\
\mu_{n} & \mu_{n-1} & \cdots & \mu_{0}
\end{array}\right|, \quad n \geq 1 .
$$

We consider the sequence of polynomials $\left\{Q_{n}\right\}$ that satisfies

$$
\mathcal{M}\left[z^{-s} Q_{n}(z)\right]=\rho_{n} \delta_{n, s}, \quad 0 \leq s \leq n, \quad n \geq 1,
$$

where $Q_{n}$, for any $n \geq 0$, is a monic polynomial of degree $n$. If the moment functional $\mathcal{M}$ is such that $D_{n} \neq 0, n \geq 0$, then we will refer to it as a semi-definite

Received by the editors September 7, 2010 and, in revised form, February 11, 2011.

2010 Mathematics Subject Classification. Primary 33D15, 42C05; Secondary 33D45.

Key words and phrases. Basic hypergeometric functions, continued fractions, orthogonal Laurent polynomials, Szegö polynomials.

This work was partially support by the joint project CAPES(Brazil)/DGU(Spain).

The second author's work was also supported by the European Community fund FEDER.

The third and fourth authors have also received other funds from CNPq, CAPES and FAPESP of Brazil for this work. 
moment functional. In this case it is easily seen that the sequence of polynomials $\left\{Q_{n}\right\}$ exists uniquely and that

$$
Q_{n}(z)=\frac{1}{D_{n-1}}\left|\begin{array}{cccc}
\mu_{0} & \mu_{-1} & \cdots & \mu_{-n} \\
\mu_{1} & \mu_{0} & \cdots & \mu_{-n+1} \\
\vdots & \vdots & & \vdots \\
\mu_{n-1} & \mu_{n-2} & \cdots & \mu_{-1} \\
1 & z & \cdots & z^{n}
\end{array}\right| \text { and } \rho_{n}=\mathcal{M}\left[z^{-n} Q_{n}(z)\right]=\frac{D_{n}}{D_{n-1}}
$$

There have been different nomenclatures used with respect to such polynomials in recent years. The polynomials $Q_{n}$ are related to orthogonal Laurent polynomials considered by, for example, Hendriksen and van Rossum [11 and Jones and Thron [15, in the sense that the Laurent polynomials

$$
B_{2 n}(z)=z^{-n} Q_{2 n}(z), \quad B_{2 n+1}(z)=z^{-n} Q_{2 n+1}(z), \quad n \geq 0,
$$

satisfy the orthogonality relations $\mathcal{M}\left[B_{n}(z) B_{m}(z)\right]=\delta_{n, m} \tilde{\rho}_{n}, n, m=0,1,2, \ldots$.

With the monic polynomials $\left\{\hat{Q}_{n}\right\}$ given by

$$
\hat{Q}_{n}(z)=\frac{1}{D_{n-1}}\left|\begin{array}{cccc}
\mu_{0} & \mu_{1} & \cdots & \mu_{n} \\
\mu_{-1} & \mu_{0} & \cdots & \mu_{n-1} \\
\vdots & \vdots & & \vdots \\
\mu_{-n+1} & \mu_{-n+2} & \cdots & \mu_{1} \\
1 & z & \cdots & z^{n}
\end{array}\right|, \quad n \geq 1
$$

we obtain the biorthogonality relations $\mathcal{M}\left[\hat{Q}_{m}(1 / z) Q_{n}(z)\right]=\delta_{n, m} \rho_{n}, \quad n, m=$ $0,1,2, \ldots$ Hence, Zhedanov 32 calls such polynomials Laurent biorthogonal.

With respect to the moment functional $\mathcal{L}\left[z^{n}\right]=\mathcal{M}\left[z^{-n}\right]=\mu_{n}, n=0, \pm 1, \pm 2, \ldots$, the reciprocal polynomials $Q_{n}^{\bullet}(z)=z^{n} Q_{n}(1 / z)$ satisfy the orthogonality relations $\mathcal{L}\left[z^{-n+s} Q_{n}^{\bullet}(z)\right]=\delta_{n, s} \rho_{n}, \quad 0 \leq s \leq n$. Polynomials satisfying such orthogonality relations have been referred to as $L$-orthogonal polynomials in some earlier contributions, including [1, of one of the present authors. We remark that Zhedanov [32] uses the definition $\mathcal{L}\left[z^{n}\right]=\mu_{n}, n=0, \pm 1, \pm 2, \ldots$, for his moment functional and $\mathcal{L}\left[\hat{Q}_{m}(z) Q_{n}(1 / z)\right]=\delta_{n, m} \rho_{n}, \quad n, m=0,1,2, \ldots$.

In a recent manuscript [17], $\left\{Q_{n}\right\}$ has been called a sequence of monic Szegö type polynomials when $\mathcal{M}$ is such that $D_{n} \neq 0$ and $\mu_{-n}=\mu_{n}$ for $n \geq 0$. In this case the Zhedanov [32] biorthogonality can be written as $\mathcal{M}\left[Q_{m}(1 / z) Q_{n}(z)\right]=\delta_{n, m} \rho_{n}$, $n, m=0,1,2, \ldots$.

However, if $\mathcal{M}$ is such that $D_{n}>0$ and $\mu_{-n}=\bar{\mu}_{n}, n \geq 0$, then this moment functional is known as a positive definite moment functional and the sequence of polynomials $\left\{S_{n}\right\}=\left\{Q_{n}\right\}$ are known as monic Szegö polynomials. Now we must have $\mathcal{M}[f]=\int_{\mathcal{C}} f(z) d \mu(z)$, where $\mu(z)=\mu\left(e^{i \theta}\right)$ is a positive measure on the unit circle $\mathcal{C}=\left\{z=e^{i \theta}: 0 \leq \theta \leq 2 \pi\right\}$. Since the integration is along the unit circle, $\int_{\mathcal{C}} z^{-j} S_{n}(z) d \mu(z)=\int_{\mathcal{C}} \bar{z}^{j} S_{n}(z) d \mu(z)$ and the associated sequence of monic Szego" polynomials $\left\{S_{n}\right\}$ are usually defined by

$$
\int_{\mathcal{C}} \overline{S_{m}(z)} S_{n}(z) d \mu(z)=\int_{0}^{2 \pi} \overline{S_{m}\left(e^{i \theta}\right)} S_{n}\left(e^{i \theta}\right) d \mu\left(e^{i \theta}\right)=\kappa_{n}^{-2} \delta_{n j}, \quad m, n=0,1,2, \ldots,
$$

where $\kappa_{n}^{-2}=\left\|S_{n}\right\|^{2}=\int_{\mathcal{C}}\left|S_{n}(z)\right|^{2} d \mu(z)$.

With his publications [28] and 29], Szegö introduced these orthogonal polynomials on the unit circle in the early 20th century. Many interesting results on these 
polynomials can be found in his classical book [30, the first edition of which was published in 1939. Since then, these polynomials which bear the name of Szegö were extensively studied by many. We cite, for example, [5], [6], 7], 10, [19], 20], 222, 25] and 27] as some of the very recent contributions. The recent publications of the two excellent volumes 23 and 24 by Simon have given a boost to the interest in studying these polynomials. We also cite the recent book 13 by Ismail containing a nice chapter on these orthogonal polynomials on the unit circle.

Some information on the Szegö polynomials with respect to the measure $d \mu\left(e^{i \theta}\right)$ $=\left[e^{-\theta}\right]^{\eta}\left[\sin ^{2}(\theta / 2)\right]^{\lambda} d \theta$, defined for $\eta, \lambda \in \mathbb{R}$ and $\lambda>-1 / 2$, are provided in [27. It was shown that these Szegö polynomials are constant multiples of the hypergeometric polynomials ${ }_{2} F_{1}(-n, b+1 ; b+\bar{b}+1 ; 1-z), n \geq 1$, where $\eta=\mathcal{I} m(b)$ and $\lambda=\mathcal{R} e(b)$.

Results used in 27] have an important root in the paper [11] of Hendriksen and van Rossum, where these authors look at T-fractions and orthogonal Laurent polynomials originating from three-term contiguous relations satisfied by the hypergeometric functions ${ }_{2} F_{1}(a, b ; c ; z)$.

In this paper, using a three-term contiguous relation satisfied by $q$-hypergeometric functions ${ }_{2} \Phi_{1}\left(q^{a}, q^{b} ; q^{c} ; q, z\right)$, we obtain information on the three-parameter class of orthogonal Laurent polynomials $\left\{z^{-\lfloor n / 2\rfloor} Q_{n}^{(b, c, d)}(z)\right\}_{n=0}^{\infty}$ on the unit circle $\mathcal{C}=\left\{z=e^{i \theta}: 0<\theta<2 \pi\right\}$, where the monic polynomials $Q_{n}^{(b, c, d)}, n \geq 0$, are given by

$$
Q_{n}^{(b, c, d)}(z)=\frac{\left(q^{c-b+1} ; q\right)_{n}}{\left(q^{b+1} ; q\right)_{n}} q^{n(b-d+1)}{ }_{2} \Phi_{1}\left(q^{-n}, q^{b+1} ; q^{-c+b-n} ; q, q^{-c+d-1} z\right),
$$

with $0<q<1$ and the three complex parameters $b, c$ and $d$ are such that $b \neq$ $-1,-2, \ldots, \quad c-b+1 \neq-1,-2, \ldots, \mathcal{R} e(d)>0$ and $\mathcal{R} e(c+2-d)>0$. The orthogonality is with respect to the semi-definite moment functional $\mathcal{M}^{(b, c, d)}$ given by

$$
\mathcal{M}^{(b, c, d)}[f(z)]=\frac{\tau^{(b, c)}}{2 \pi} \int_{0}^{2 \pi} f\left(e^{i \theta}\right) \frac{\left(q^{-b+d} e^{i \theta} ; q\right)_{\infty}\left(q^{b-d+1} e^{-i \theta} ; q\right)_{\infty}}{\left(q^{d} e^{i \theta} ; q\right)_{\infty}\left(q^{c+2-d} e^{-i \theta} ; q\right)_{\infty}} d \theta
$$

Here the constant $\tau^{(b, c)}$, defined in Theorem 3.5, is such that $\mathcal{M}^{(b, c, d)}[1]=1$. By considering separately the real and imaginary parts of $b, c$ and $d$, and neglecting $\mathcal{I} m(d)$, which induces only a rotation, we can also consider $\left\{z^{-\lfloor n / 2\rfloor} Q_{n}^{(b, c, d)}(z)\right\}_{n=0}^{\infty}$ as a five-real-parameter class of orthogonal Laurent polynomials.

The class of polynomials considered here is somewhat different and broader than the class of orthogonal Laurent polynomials $\left\{z^{-\lfloor n / 2\rfloor} P_{n}(-z, \alpha, \beta)\right\}_{n=0}^{\infty}$ that follows from Pastro 21, where

$$
P_{n}(z, \alpha, \beta)={ }_{2} \Phi_{1}\left(\tilde{q}^{-n}, \tilde{q}^{\alpha} ; \tilde{q}^{2-\beta-n} ; \tilde{q}, \tilde{q}^{-\beta+3 / 2} z\right),
$$

with $|\tilde{q}|<1, \alpha>1 / 2$ and $\beta>1 / 2$. Pastro shows that the polynomials $P_{n}(-z, \alpha, \beta)$ are the Laurent biorthogonal polynomials with respect to the semi-definite moment functional given by

$$
\tilde{\mathcal{M}}^{(\alpha, \beta)}[f(z)]=\int_{0}^{2 \pi} f\left(e^{i \theta}\right) \frac{\left(\tilde{q}^{1 / 2} e^{i \theta} ; \tilde{q}\right)_{\infty}\left(\tilde{q}^{1 / 2} e^{-i \theta} ; \tilde{q}\right)_{\infty}}{\left(\tilde{q}^{\alpha-1 / 2} e^{i \theta} ; \tilde{q}\right)_{\infty}\left(\tilde{q}^{\beta-1 / 2} e^{-i \theta} ; \tilde{q}\right)_{\infty}} d \theta
$$

These Pastro polynomials can be considered as belonging to a class with the three real parameters $\alpha, \beta$ and, say, $\vartheta$ if one assumes the $\tilde{q}$ to be such that $\tilde{q}=|\tilde{q}| e^{i \vartheta}$. An 
explicit expression for the moments $\tilde{\mathcal{M}}^{(\alpha, \beta)}\left[z^{-n}\right]$ is found in Vinet and Zhedanov 31 .

Note that the moment functional $\tilde{\mathcal{M}}^{(\alpha, \beta)}$ can only be made positive definite with the choice $-1<\tilde{q}<1$ and $\alpha=\beta>1 / 2$. Thus with this choice, Pastro 21] recovers the class of real Szegó polynomials previously described by Askey [4, p. 806].

By a special choice of the parameters $b, c$ and $d$ we also obtain in the present manuscript information regarding the class of (complex and real) Szegö polynomials $S_{n}^{(\lambda, \eta, \phi)}$ characterized by the reflection coefficients

$$
a_{n}^{(\lambda, \eta, \phi)}=\frac{\left(q^{\lambda+i \eta} ; q\right)_{n}}{\left(q^{\lambda+1-i \eta} ; q\right)_{n}} q^{n\left[\frac{1}{2}-i(\eta+\phi)\right]}, \quad n \geq 1,
$$

where $\lambda, \eta, \phi \in \mathbb{R}$ and $\lambda>-1 / 2$. The parameter $\phi$, which comes from $\mathcal{I} m(d)$, induces only a rotation and can be made equal to zero without any loss of generality.

The polynomials obtained by taking $\eta=\phi=0$ and $\lambda>-1 / 2$, for example, coincide with the real Szegö polynomials of [21] and [4, obtained when $0<\tilde{q}<1$ and $\alpha=\beta>1 / 2$.

The paper is organized as follows. In Section 2 we present some fundamental results on three-term recurrence relations, continued fractions and basic hypergeometric functions, which we will be using in later sections. In Section 3 we define the monic $q$-hypergeometric polynomials $Q_{n}^{(b, c, d)}(z)$ and obtain their orthogonality and asymptotic properties. In Section 4 , in addition to discussing when the polynomials $Q_{n}^{(b, c, d)}(z)$ coincide with the Szegö polynomials $S_{n}^{(\lambda, \eta, \phi)}$ mentioned above, we also obtain explicitly the associated Szegö function.

\section{SOME PRELIMINARY RESUlts}

Let $\left\{Q_{n}\right\}$ be the sequence of polynomials given by the three-term recurrence relation

$$
Q_{n+1}(z)=\left(z+\beta_{n+1}\right) Q_{n}(z)-\alpha_{n+1} z Q_{n-1}(z), \quad n \geq 1,
$$

with $Q_{0}(z)=1$ and $Q_{1}(z)=z+\beta_{1}$.

Lemma 2.1. Let $\beta_{1} \neq 0$ and $\alpha_{n+1} \neq 0$ for $n \geq 1$. Given any sequence $\left\{h_{n}\right\}$ of arbitrary complex numbers $h_{n}$ (or complex functions $h_{n}(z)$ ), let the sequence of functions $\left\{G_{n}\left(h_{n} ; z\right)\right\}$ be such that $G_{1}\left(h_{1} ; z\right)=\frac{\beta_{1}}{z+\beta_{1}-h_{1}}$ and

$$
G_{n}\left(h_{n} ; z\right)=\frac{\beta_{1}}{\mid z+\beta_{1}}-\frac{\alpha_{2} z}{2 z+\beta_{2}}-\cdots-\frac{\alpha_{n} z}{\mid z+\beta_{n}-h_{n}}, \quad n \geq 2 .
$$

Then

$$
G_{n}\left(h_{n} ; z\right)-G_{n}(0 ; z)=\frac{\beta_{1} \alpha_{2} \alpha_{3} \cdots \alpha_{n} h_{n} z^{n-1}}{Q_{n}(z)\left[Q_{n}(z)-h_{n} Q_{n-1}(z)\right]} .
$$

Proof. Let the sequence of polynomials $\left\{R_{n}\right\}$ be such that

$$
R_{n+1}(z)=\left(z+\beta_{n+1}\right) R_{n}(z)-\alpha_{n+1} z R_{n-1}(z), \quad n \geq 1,
$$

with $R_{0}(z)=0, R_{1}(z)=\beta_{1}$. Then from basic results on continued fractions (see, for example, [14, 18])

$$
G_{n}\left(h_{n} ; z\right)-G_{n}(0 ; z)=\frac{R_{n}(z)-h_{n} R_{n-1}(z)}{Q_{n}(z)-h_{n} Q_{n-1}(z)}-\frac{R_{n}(z)}{Q_{n}(z)}, \quad n \geq 1 .
$$


Hence,

$$
G_{n}\left(h_{n} ; z\right)-G_{n}(0 ; z)=\frac{h_{n}\left[R_{n}(z) Q_{n-1}(z)-Q_{n}(z) R_{n-1}(z)\right]}{Q_{n}(z)\left[Q_{n}(z)-h_{n} Q_{n-1}(z)\right]}, \quad n \geq 1 .
$$

Therefore, the lemma follows from $R_{n}(z) Q_{n-1}(z)-Q_{n}(z) R_{n-1}(z)=\beta_{1} \alpha_{2} \alpha_{3} \ldots$ $\alpha_{n} z^{n-1}$.

As a particular case of this lemma, if one takes $h_{n}=\alpha_{n+1} z /\left(z+\beta_{n+1}\right)$, then

$$
G_{n+1}(0 ; z)-G_{n}(0 ; z)=\frac{\beta_{1} \alpha_{2} \alpha_{3} \cdots \alpha_{n+1}}{Q_{n}(z) Q_{n+1}(z)} z^{n}, \quad n \geq 1 .
$$

Lemma 2.2. In the three-term recurrence relation (2.1), if

$$
\beta_{n} \neq 0 \quad \text { and } \quad \alpha_{n+1} \neq 0, \quad n \geq 1,
$$

then there exists a semi-definite moment functional $\mathcal{M}$ such that the polynomials $Q_{n}$ satisfy

$$
\mathcal{M}\left[z^{-s} Q_{n}(z)\right]=\delta_{n, s} \rho_{n}, \quad 0 \leq s \leq n, \quad n \geq 1,
$$

where $\rho_{n}=\frac{\alpha_{2} \cdots \alpha_{n+1}}{\beta_{2} \cdots \beta_{n+1}}$. Moreover, the associated moments $\mu_{n}=\mathcal{M}\left[z^{-n}\right], n=$ $0, \pm 1, \pm 2, \ldots$ are such that $L_{0}(z)=\sum_{j=0}^{\infty} \mu_{j} z^{j}, L_{\infty}(z)=-\sum_{j=1}^{\infty} \mu_{-j} z^{-j}$, where

$$
\begin{aligned}
L_{0}(z)-G_{n}(0 ; z) & =\rho_{n} \frac{1}{Q_{n}(0)} z^{n}+O\left(z^{n+1}\right), \\
L_{\infty}(z)-G_{n}(0 ; z) & =\rho_{n} Q_{n+1}(0) \frac{1}{z^{n+1}}+O\left(\frac{1}{z^{n+2}}\right) .
\end{aligned}
$$

Proof. First note that $Q_{n}(0)=\beta_{1} \beta_{2} \cdots \beta_{n} \neq 0$. Now from (2.2) by considering the expansions of $G_{n}(0 ; z)$ about the origin and infinity there exist power series $L_{0}(z)=\sum_{j=0}^{\infty} \mu_{j} z^{j}$ and $L_{\infty}(z)=-\sum_{j=1}^{\infty} \mu_{-j} z^{-j}$ such that (2.3) holds.

With respect to these power series coefficients, if we define the moment functional $\mathcal{M}$ by (1.1), then the lemma follows from the linear system on the coefficients of $Q_{n}$ and $R_{n}$ obtained from (2.3).

For $a, b, c \in \mathbb{C}, c \neq 0,-1,-2, \ldots$ and $0<|q|<1$, the ${ }_{2} \Phi_{1} q$-hypergeometric or the ${ }_{2} \Phi_{1}$ basic hypergeometric function (hypergeometric function with base $q$ ) may be defined by

$$
{ }_{2} \Phi_{1}\left(q^{a}, q^{b} ; q^{c} ; q, z\right)=\sum_{n=0}^{\infty} \frac{\left(q^{a} ; q\right)_{n}\left(q^{b} ; q\right)_{n}}{\left(q^{c} ; q\right)_{n}(q ; q)_{n}} z^{n},
$$

for $|z|<1$ and by analytic continuation for other values of $z \in \mathbb{C}$. Here, $\left(q^{a} ; q\right)_{0}=1$ and $\left(q^{a} ; q\right)_{n}=\left(1-q^{a}\right)\left(1-q^{a+1}\right) \cdots\left(1-q^{a+n-1}\right), n \geq 1$.

For more information regarding $q$-hypergeometric functions, we refer to, for example, Andrews, Askey and Roy [2], Gasper and Rahman [8], Koekoek and Swarttouw [16] and Slater [26].

Two "distinct" $q$-hypergeometric functions ${ }_{2} \Phi_{1}\left(q^{a_{1}}, q^{a_{2}} ; q^{a_{3}} ; q, z\right)$ and ${ }_{2} \Phi_{1}\left(q^{\tilde{a}_{1}}\right.$, $\left.q^{\tilde{a}_{2}} ; q^{\tilde{a}_{3}} ; q, z\right)$ may be called contiguous if $\left|a_{i}-\tilde{a}_{i}\right|=0$ or 1 for at least one $i \in$ $\{1,2,3\}$. There are interesting relations between contiguous $q$-hypergeometric functions called contiguous relations. 
Lemma 2.3. If $c \neq 0,-1,-2, \ldots$, then

$$
\begin{aligned}
{ }_{2} \Phi_{1}\left(q^{a}, q^{b+1} ; q^{c} ; q, z\right)=(1 & \left.+\frac{1-q^{a-b}}{1-q^{c}} q^{b} z\right){ }_{2} \Phi_{1}\left(q^{a+1}, q^{b+1} ; q^{c+1} ; q, z\right) \\
& -\frac{\left(1-q^{a+1}\right)\left(1-q^{c-b}\right)}{\left(1-q^{c}\right)\left(1-q^{c+1}\right)} q^{b} z{ }_{2} \Phi_{1}\left(q^{a+2}, q^{b+1} ; q^{c+2} ; q, z\right) .
\end{aligned}
$$

Proof. From contiguous relations obtained by Heine (see [8, p. 22]), we consider the following:

$$
\begin{aligned}
{ }_{2} \Phi_{1}\left(q^{a+1}, q^{b+1} ; q^{c+1} ; q, z\right)= & { }_{2} \Phi_{1}\left(q^{a+1}, q^{b} ; q^{c} ; q, z\right) \\
& +\frac{\left(1-q^{a+1}\right)\left(1-q^{c-b}\right)}{\left(1-q^{c}\right)\left(1-q^{c+1}\right)} q^{b} z{ }_{2} \Phi_{1}\left(q^{a+2}, q^{b+1} ; q^{c+2} ; q, z\right), \\
{ }_{2} \Phi_{1}\left(q^{a+1}, q^{b} ; q^{c} ; q, z\right)= & { }_{2} \Phi_{1}\left(q^{a}, q^{b+1} ; q^{c} ; q, z\right) \\
& -\frac{\left(1-q^{a-b}\right)}{\left(1-q^{c}\right)} q^{b} z{ }_{2} \Phi_{1}\left(q^{a+1}, q^{b+1} ; q^{c+1} ; q, z\right),
\end{aligned}
$$

which hold for $c \neq 0,-1,-2, \ldots$ Substitution for ${ }_{2} \Phi_{1}\left(q^{a+1}, q^{b} ; q^{c} ; q, z\right)$ in the first relation using the other gives the required result.

We will be using the $q$-binomial theorem (see [16, Eq. (0.5.2)])

$$
{ }_{2} \Phi_{1}\left(q^{a}, q^{c} ; q^{c} ; q, z\right)={ }_{1} \Phi_{0}\left(q^{a} ; q, z\right)=\frac{\left(q^{a} z ; q\right)_{\infty}}{(z ; q)_{\infty}},
$$

which holds for $c \neq 0,-1,-2, \ldots$ and $|z|<1$, and the Heine transformation formula (see [16, Eq. (0.6.3)])

$$
{ }_{2} \Phi_{1}\left(q^{a}, q^{b} ; q^{c} ; q, z\right)=\frac{\left(q^{a+b-c} z ; q\right)_{\infty}}{(z ; q)_{\infty}}{ }_{2} \Phi_{1}\left(q^{-a+c}, q^{-b+c} ; q^{c} ; q, q^{a+b-c} z\right),
$$

which holds for $c \neq 0,-1,-2, \ldots$ and $|z|<\min \left\{1,\left|q^{c-a-b}\right|\right\}$. We will also be needing the polynomial identities (see [16, Eq. (0.6.19)])

$$
\begin{aligned}
& { }_{2} \Phi_{1}\left(q^{-n}, q^{b} ; q^{c} ; q, z\right) \\
& =\frac{\left(q^{b} ; q\right)_{n}}{\left(q^{c} ; q\right)_{n}} q^{-n(n+1) / 2}(-z)_{2}^{n} \Phi_{1}\left(q^{-n}, q^{-c-n+1} ; q^{-b-n+1} ; q, q^{c-b+n+1} z^{-1}\right),
\end{aligned}
$$

for $n \geq 0$, which hold when $c \neq 0,-1,-2, \ldots$ and $b \neq-n+1,-n+2,-n+3, \ldots$.

\section{3. $q$-ORTHOGONAL LAURENT POLYNOMIALS}

From now on we restrict the value of $q$ to be such that $0<q<1$. Then for any $b \in \mathbb{C}$ we have

$$
\overline{q^{b}}=q^{\bar{b}} \quad \text { and } \quad\left|q^{b}\right|=q^{\mathcal{R} e(b)} .
$$

With $b, c, d \in \mathbb{C}$ and $c-b+1 \neq 0,-1,-2, \ldots$, let

$$
F_{n}^{(b, c, d)}(z)=\frac{{ }_{2} \Phi_{1}\left(q^{n+1}, q^{-b} ; q^{c-b+n+2} ; q, q^{d} z\right)}{{ }_{2} \Phi_{1}\left(q^{n}, q^{-b} ; q^{c-b+n+1} ; q, q^{d} z\right)}, \quad n \geq 0 .
$$

Then from Lemma 2.3.

$$
F_{n}^{(b, c, d)}(z)=\frac{1}{1+g_{n+1}^{(b, c, d)} z-f_{n+2}^{(b, c, d)} z F_{n+1}^{(b, c, d)}(z)}, \quad n \geq 0,
$$


where

$$
g_{n}^{(b, c, d)}=\frac{1-q^{b+n}}{1-q^{c-b+n}} q^{-b+d-1}, \quad f_{n+1}^{(b, c, d)}=\frac{\left(1-q^{n}\right)\left(1-q^{c+n+1}\right)}{\left(1-q^{c-b+n}\right)\left(1-q^{c-b+n+1}\right)} q^{-b+d-1},
$$

for $n \geq 1$. This leads to the continued fraction expansion

$$
\begin{aligned}
& F_{0}^{(b, c, d)}(z)= \\
& \frac{1}{1_{1+g_{1}^{(b, c, d)} z}}-\frac{f_{2}^{(b, c, d)} z}{1+g_{2}^{(b, c, d)} z}-\cdots-\frac{f_{n}^{(b, c, d)} z}{\sqrt{1+g_{n}^{(b, c, d)} z-f_{n+1}^{(b, c, d)} z F_{n}^{(b, c, d)}(z)}} .
\end{aligned}
$$

Also assuming $b \neq-1,-2, \ldots$, this can be written in the equivalent form

$$
\begin{aligned}
& F_{0}^{(b, c, d)}(z)= \\
& \quad \beta_{1}^{(b, c, d)} J_{2}^{(b, c, d)}-\frac{\alpha_{2}^{(b, c, d)} z}{z+\beta_{2}^{(b, c, d)}}-\cdots-\frac{\alpha_{n}^{(b, c, d)} z}{z+\beta_{n}^{(b, c, d)}}-\frac{\alpha_{n+1}^{(b, c, d)} z F_{n}^{(b, c, d)}(z)}{\beta_{n+1}^{(b, c, d)}},
\end{aligned}
$$

where $\beta_{n}^{(b, c, d)}=1 / g_{n}^{(b, c, d)}$ and $\alpha_{n+1}^{(b, c, d)}=f_{n+1}^{(b, c, d)} /\left(g_{n}^{(b, c, d)} g_{n+1}^{(b, c, d)}\right), n \geq 1$.

Theorem 3.1. With $b \neq-1,-2, \ldots$ and $c-b+1 \neq-1,-2, \ldots$, let the sequence of monic polynomials $\left\{Q_{n}^{(b, c, d)}\right\}$ be given by

$$
Q_{n+1}^{(b, c, d)}(z)=\left(z+\beta_{n+1}^{(b, c, d)}\right) Q_{n}^{(b, c, d)}(z)-\alpha_{n+1}^{(b, c, d)} z Q_{n-1}^{(b, c, d)}(z), \quad n \geq 1,
$$

with $Q_{0}^{(b, c, d)}(z)=1$ and $Q_{1}^{(b, c, d)}(z)=z+\beta_{1}^{(b, c, d)}$, where

$$
\beta_{n}^{(b, c, d)}=\frac{1-q^{c-b+n}}{1-q^{b+n}} q^{b-d+1}, \quad \alpha_{n+1}^{(b, c, d)}=\frac{\left(1-q^{n}\right)\left(1-q^{c+n+1}\right)}{\left(1-q^{b+n}\right)\left(1-q^{b+n+1}\right)} q^{b-d+1}, \quad n \geq 1 .
$$

Then the polynomials $Q_{n}^{(b, c, d)}$ satisfy the orthogonality relations

$$
\mathcal{M}^{(b, c, d)}\left[z^{-s} Q_{n}^{(b, c, d)}(z)\right]=\delta_{n, s} \rho_{n}^{(b, c)}, \quad 0 \leq s \leq n, \quad n \geq 1,
$$

with respect to the semi-definite moment functional

$$
\mathcal{M}^{(b, c, d)}\left[z^{-j}\right]=\frac{\left(q^{-b} ; q\right)_{j}}{\left(q^{c-b+2} ; q\right)_{j}} q^{j d}, \quad j=0, \pm 1, \pm 2, \ldots
$$

Here,

$$
\rho_{n}^{(b, c)}=\frac{\alpha_{2}^{(b, c, d)} \cdots \alpha_{n+1}^{(b, c, d)}}{\beta_{2}^{(b, c, d)} \cdots \beta_{n+1}^{(b, c, d)}}=\frac{(q ; q)_{n}\left(q^{c+2} ; q\right)_{n}}{\left(q^{b+1} ; q\right)_{n}\left(q^{c-b+2} ; q\right)_{n}} .
$$

Proof. We first prove the theorem for $c-b+1 \neq 0,-1,-2, \ldots$ and $b \neq-1,-2, \ldots$. With these restrictions $\beta_{n}^{(b, c, d)} \neq 0$ and $\alpha_{n+1}^{(b, c, d)} \neq 0, n \geq 1$, and hence from Lemma 2.2 there exists a semi-definite moment functional such that (3.4) holds.

To obtain the values of $\mu_{j}^{(b, c, d)}=\mathcal{M}^{(b, c, d)}\left[z^{-j}\right], j=0, \pm 1, \pm 2, \ldots$, let us consider the functions

$$
G_{n}^{(b, c, d)}(z)=\frac{\beta_{1}^{(b, c, d)} \mid}{\mid z+\beta_{1}^{(b, c, d)}}-\frac{\alpha_{2}^{(b, c, d)} z}{\mid z+\beta_{2}^{(b, c, d)}}-\cdots-\frac{\alpha_{n}^{(b, c, d)} z}{z+\beta_{n}^{(b, c, d)}}, \quad n \geq 1 .
$$


Then from Lemma 2.1 and the continued fraction expansion (3.2),

$$
\begin{aligned}
F_{0}^{(b, c, d)}(z) & -G_{n}^{(b, c, d)}(z) \\
= & \frac{\beta_{1}^{(b, c, d)} \alpha_{2}^{(b, c, d)} \cdots \alpha_{n}^{(b, c, d)} \alpha_{n+1}^{(b, c, d)} z^{n} F_{n}^{(b, c, d)}(z)}{Q_{n}^{(b, c, d)}(z)\left[\beta_{n+1}^{(b, c, d)} Q_{n}^{(b, c, d)}(z)-\alpha_{n+1}^{(b, c, d)} z F_{n}^{(b, c, d)}(z) Q_{n-1}^{(b, c, d)}(z)\right]} \\
= & \rho_{n}^{(b, c)} \frac{1}{Q_{n}^{(b, c, d)}(0)} z^{n}+O\left(z^{n+1}\right), \quad \text { for } \quad n \geq 1 .
\end{aligned}
$$

Since $F_{0}^{(b, c, d)}(z)={ }_{2} \Phi_{1}\left(q, q^{-b} ; q^{c-b+2} ; q, q^{d} z\right)$, from the latter part of Lemma 2.2.

$$
\mu_{j}^{(b, c, d)}=\frac{\left(q^{-b} ; q\right)_{j}}{\left(q^{c-b+2} ; q\right)_{j}} q^{j d}, \quad j=0,1,2, \ldots,
$$

thus giving the results for the positive moments.

From (3.1), by realizing that $g_{n}^{(c-b, c, c+2-d)}=\beta_{n}^{(b, c, d)}$ and $f_{n+1}^{(c-b, c, c+2-d)}=\alpha_{n+1}^{(b, c, d)}$, $n \geq 1$, we also obtain the continued fraction expansion

$$
\begin{aligned}
& \frac{\beta_{1}^{(b, c, d)}}{z} F_{0}^{(c-b, c, c+2-d)}\left(z^{-1}\right)= \\
& \beta_{1}^{(b, c, d)} \mid \begin{array}{c}
\alpha_{2}^{(b, c, d)} z \\
z+\beta_{1}^{(b, c, d)}-\cdots-\frac{\alpha_{n}^{(b, c, d)} z}{\mid z+\beta_{2}^{(b, c, d)}}-\cdots-\alpha_{n}^{(b, c, d)}
\end{array}-\frac{\alpha_{n+1}^{(b, c, d)} F_{n}^{(c-b, c, c+2-d)}\left(z^{-1}\right)}{\mid z+} 1 .
\end{aligned}
$$

Hence, again from Lemma 2.1 ,

$$
\begin{aligned}
\frac{\beta_{1}^{(b, c, d)}}{z} F_{0}^{(c-b, c, c+2-d)}\left(z^{-1}\right)-G_{n}^{(b, c, d)}(z) & \\
= & \frac{\beta_{1}^{(b, c, d)} \alpha_{2}^{(b, c, d)} \cdots \alpha_{n}^{(b, c, d)} \alpha_{n+1}^{(b, c, d)} z^{n-1} F_{n}^{(c-b, c, c+2-d)}\left(z^{-1}\right)}{Q_{n}^{(b, c, d)}(z)\left[Q_{n}^{(b, c, d)}(z)-\alpha_{n+1}^{(b, c, d)} F_{n}^{(c-b, c, c+2-d)}\left(z^{-1}\right) Q_{n-1}^{(b, c, d)}(z)\right]} \\
= & \rho_{n}^{(b, c)} Q_{n+1}^{(b, c, d)}(0) \frac{1}{z^{n+1}}+O\left(\frac{1}{z^{n+2}}\right), \quad \text { for } \quad n \geq 1 .
\end{aligned}
$$

Since $F_{0}^{(c-b, c, c+2-d)}\left(z^{-1}\right)={ }_{2} \Phi_{1}\left(q, q^{-c+b} ; q^{b+2} ; q, q^{c+2-d} z^{-1}\right)$, from the latter part of Lemma 2.2.

$$
\mu_{-j}^{(b, c, d)}=\frac{\left(q^{-c+b-1} ; q\right)_{j}}{\left(q^{b+1} ; q\right)_{j}} q^{j(c+2-d)}, \quad j=1,2,3, \ldots .
$$

Thus, using $(a, q)_{n}=(a ; q)_{\infty} /\left(a q^{n} ; q\right)_{\infty}$, for $n=0, \pm 1, \pm 2, \ldots$, we also obtain the results for the negative moments. This concludes the theorem for $c-b+1 \neq$ $0,-1,-2, \ldots$ and $b \neq-1,-2, \ldots$.

Now to extend the results for $c-b+1 \neq-1,-2, \ldots$ and $b \neq-1,-2, \ldots$, we need to prove the theorem for $c-b+1=0$ and $b \neq-1,-2, \ldots$.

If $b \neq-1,-2, \ldots$, then $\beta_{1}^{(b, b-1, d)}=0$ and $\beta_{n+1}^{(b, b-1, d)}=\alpha_{n+1}^{(b, b-1, d)} \neq 0$ for $n \geq 1$. Hence, $Q_{n}^{(b, b-1, d)}(z)=z^{n}, n \geq 0$ and

$$
\mathcal{M}^{(b, b-1, d)}\left[z^{-s} Q_{n}^{(b, b-1, d)}(z)\right]=\mathcal{M}^{(b, b-1, d)}\left[z^{n-s}\right]=\frac{\left(q^{-b} ; q\right)_{-n+s}}{(q ; q)_{-n+s}} q^{(-n+s) d} .
$$

Since

$$
\frac{\left(q^{-b} ; q\right)_{-n+s}}{(q ; q)_{-n+s}} q^{(-n+s) d}=0 \quad \text { if } s<n \quad \text { and } \quad \rho_{n}^{(b, b-1)}=\frac{\left(q^{-b} ; q\right)_{0}}{(q ; q)_{0}} q^{(0) d}=1
$$


the validity of the theorem when $c-b+1=0$ and $b \neq-1,-2, \ldots$ is confirmed. This concludes the theorem.

The same explicit expression for the moments, when the moment functional is considered as in Pastro [21, is obtained in 31.

From the three-term recurrence relation (3.3) it follows that

$$
Q_{n}^{(b, c, d)}(0)=\beta_{1}^{(b, c, d)} \beta_{2}^{(b, c, d)} \cdots \beta_{n}^{(b, c, d)}=\frac{\left(q^{c-b+1} ; q\right)_{n}}{\left(q^{b+1} ; q\right)_{n}} q^{n(b-d+1)}, \quad n \geq 1 .
$$

Theorem 3.2. Let $b \neq-1,-2, \ldots$ and $c-b+1 \neq-1,-2, \ldots$ Then

$$
\begin{aligned}
& \text { a) } \lim _{n \rightarrow \infty} \beta_{n}^{(b, c, d)}=q^{b-d+1}, \quad \lim _{n \rightarrow \infty} \alpha_{n}^{(b, c, d)}=q^{b-d+1}, \\
& \text { b) } \lim _{n \rightarrow \infty} q^{-n(b-d+1)} Q_{n}^{(b, c, d)}(0)=(1-q)^{-c+2 b} \frac{\Gamma_{q}(b+1)}{\Gamma_{q}(c-b+1)}, \\
& \text { c) } \lim _{n \rightarrow \infty} \rho_{n}^{(b, c)}=\frac{\Gamma_{q}(b+1) \Gamma_{q}(c-b+2)}{\Gamma_{q}(c+2)} .
\end{aligned}
$$

Proof. Part $a$ ) of this theorem is clear. To obtain parts $b$ ) and $c$ ) we use the definition

$$
\Gamma_{q}(x)=\frac{(q ; q)_{\infty}}{\left(q^{x} ; q\right)_{\infty}}(1-q)^{1-x}
$$

of the $q$-gamma function.

Theorem 3.3. Let $b \neq-1,-2, \ldots$ and $c-b+1 \neq-1,-2, \ldots$ Then the monic polynomials $Q_{n}^{(b, c, d)}, n \geq 0$, given by the recurrence relation (3.3) have the explicit representation

$$
Q_{n}^{(b, c, d)}(z)=\frac{\left(q^{c-b+1} ; q\right)_{n}}{\left(q^{b+1} ; q\right)_{n}} q^{n(b-d+1)}{ }_{2} \Phi_{1}\left(q^{-n}, q^{b+1} ; q^{-c+b-n} ; q, q^{-c+d-1} z\right)
$$

Proof. From (3.3) it is easily verified that the reciprocal (or inverse) polynomials

$$
Q_{n}^{*(b, c, d)}(z)=z^{n} \overline{Q_{n}^{(b, c, d)}(1 / \bar{z})} \quad \text { and } \quad Q_{n}^{\bullet(b, c, d)}(z)=z^{n} Q_{n}^{(b, c, d)}(1 / z), \quad n \geq 0,
$$

satisfy the three-term recurrence relations

$$
\begin{aligned}
& Q_{n+1}^{*(b, c, d)}(z)=\left(1+\beta_{n+1}^{(\bar{b}, \bar{c}, \bar{d})} z\right) Q_{n}^{*(b, c, d)}(z)-\alpha_{n+1}^{(\bar{b}, \bar{c}, \bar{d})} z Q_{n-1}^{*(b, c, d)}(z), \\
& Q_{n+1}^{\bullet(b, c, d)}(z)=\left(1+\beta_{n+1}^{(b, c, d)} z\right) Q_{n}^{\bullet(b, c, d)}(z)-\alpha_{n+1}^{(b, c, d)} z Q_{n-1}^{\bullet(b, c, d)}(z),
\end{aligned}
$$

with $Q_{0}^{*(b, c, d)}(z)=Q_{0}^{\bullet(b, c, d)}(z)=1, Q_{1}^{*(b, c, d)}(z)=1+\beta_{1}^{(\bar{b}, \bar{c}, \bar{d})} z$ and $Q_{1}^{\bullet(b, c, d)}(z)=$ $1+\beta_{1}^{(b, c, d)} z$. This means that

$$
\begin{aligned}
& Q_{n}^{*(b, c, d)}(z)={ }_{2} \Phi_{1}\left(q^{-n}, q^{\bar{c}-\bar{b}+1} ; q^{-\bar{b}-n} ; q, q^{-\bar{d}+1} z\right), \\
& Q_{n}^{\bullet(b, c, d)}(z)={ }_{2} \Phi_{1}\left(q^{-n}, q^{c-b+1} ; q^{-b-n} ; q, q^{-d+1} z\right),
\end{aligned}
$$

which we can easily verify from Lemma 2.3 . Hence, application of the transformation (2.6) in $Q_{n}^{\bullet(b, c, d)}$, for example, gives the required results of the theorem. 
Note that by the application of the transform (2.5) in $Q_{n}^{*(b, c, d)}$, for example, we can also write that

$$
\frac{\left(q^{-\bar{d}+1} z ; q\right)_{\infty}}{\left(q^{\bar{c}-\bar{d}+2} z ; q\right)_{\infty}} Q_{n}^{*(b, c, d)}(z)={ }_{2} \Phi_{1}\left(q^{-\bar{b}}, q^{-\bar{c}-n-1} ; q^{-\bar{b}-n} ; q, q^{\bar{c}-\bar{d}+2} z\right), \quad n \geq 1,
$$

provided that $|z|<q^{-\mathcal{R} e(c-d+2)}$. This can also be directly verified from Lemma 2.3 and (3.6).

Theorem 3.4. Let $b \neq-1,-2, \ldots, \quad c-b+1 \neq-1,-2, \ldots$ and

$$
\sigma=\min \left\{q^{-\mathcal{R} e(c-d+2)}, q^{-\mathcal{R} e(b-d+1)}\right\} .
$$

Then uniformly on compact subsets of $|z|<\sigma$,

$$
\lim _{n \rightarrow \infty} Q_{n}^{*(b, c, d)}(z)=\frac{\left(q^{\bar{c}-\bar{d}+2} z ; q\right)_{\infty}}{\left(q^{\bar{b}-\bar{d}+1} z ; q\right)_{\infty}} .
$$

Proof. Since

$$
\lim _{n \rightarrow \infty} \frac{\left(q^{-\bar{c}-n-1} ; q\right)_{j}}{\left(q^{-\bar{b}-n} ; q\right)_{j}} q^{(\bar{c}-\bar{d}+2) j}=q^{(\bar{b}-\bar{d}+1) j},
$$

using Lebesgue's dominated convergence theorem and then (2.4), we obtain

$\lim _{n \rightarrow \infty}{ }_{2} \Phi_{1}\left(q^{-\bar{b}}, q^{-\bar{c}-n-1} ; q^{-\bar{b}-n} ; q, q^{\bar{c}-\bar{d}+2} z\right)={ }_{1} \Phi_{0}\left(q^{-\bar{b}} ; q, q^{\bar{b}-\bar{d}+1} z\right)=\frac{\left(q^{-\bar{d}+1} z ; q\right)_{\infty}}{\left(q^{\bar{b}-\bar{d}+1} z ; q\right)_{\infty}}$, uniformly on compact subsets of $|z|<\sigma$. Thus, the result of the theorem follows.

Theorem 3.5. In addition to $b \neq-1,-2, \ldots$ and $c-b+1 \neq-1,-2, \ldots$, if one also assumes that

$$
\mathcal{R} e(c+2)>\mathcal{R} e(d)>0,
$$

then the polynomials $Q_{n}^{(b, c, d)}, n \geq 0$, given by (3.5), satisfy the orthogonality relations

$$
\frac{\tau^{(b, c)}}{2 \pi i} \int_{\mathcal{C}} z^{-s} Q_{n}^{(b, c, d)}(z) \frac{\left(q^{-b+d} z ; q\right)_{\infty}\left(q^{b-d+1} / z ; q\right)_{\infty}}{\left(q^{d} z ; q\right)_{\infty}\left(q^{c-d+2} / z ; q\right)_{\infty}} \frac{1}{z} d z=\delta_{n, s} \rho_{n}^{(b, c)}, \quad 0 \leq s \leq n .
$$

Here, $\rho_{n}^{(b, c)}$ are as in Theorem 3.1 and

$$
\tau^{(b, c)}=\frac{(q ; q)_{\infty}\left(q^{c+2} ; q\right)_{\infty}}{\left(q^{c-b+2} ; q\right)_{\infty}\left(q^{b+1} ; q\right)_{\infty}} .
$$

Proof. Let us consider the following identity of Ramanujan:

$$
\sum_{-\infty}^{\infty} \frac{(\alpha ; q)_{n}}{(\beta ; q)_{n}} x^{n}=\frac{(q ; q)_{\infty}\left(\frac{\beta}{\alpha} ; q\right)_{\infty}(\alpha x ; q)_{\infty}\left(\frac{q}{\alpha x} ; q\right)_{\infty}}{(\beta ; q)_{\infty}\left(\frac{q}{\alpha} ; q\right)_{\infty}\left(\frac{\beta}{\alpha x} ; q\right)_{\infty}(x ; q)_{\infty}},
$$

which holds for $\left|\beta \alpha^{-1}\right|<|x|<1$. Simple proofs of this identity can be found in 3 ] and [12].

In our case, since $0<q<1$, with the assumptions of the theorem if we take $x=q^{d} z, \alpha=q^{-b}$ and $\beta=q^{c-b+2}$, then

$$
\sum_{-\infty}^{\infty} \frac{\left(q^{-b} ; q\right)_{n}}{\left(q^{c-b+2} ; q\right)_{n}} q^{n d} z^{n}=\tau^{(b, c)} \frac{\left(q^{-b+d} z ; q\right)_{\infty}\left(q^{b-d+1} / z ; q\right)_{\infty}}{\left(q^{d} z ; q\right)_{\infty}\left(q^{c+2-d} / z ; q\right)_{\infty}}
$$

which holds for $\left|q^{c+2-d}\right|<|z|<\left|q^{-d}\right|$, where $\left|q^{c+2-d}\right|<1$ and $\left|q^{-d}\right|>1$. 
Hence, multiplying by $z^{-j-1}$ and integrating along the unit circle we obtain from Laurent's theorem

$\frac{\left(q^{-b} ; q\right)_{j}}{\left(q^{c-b+2} ; q\right)_{j}} q^{j d}=\frac{\tau^{(b, c)}}{2 \pi i} \int_{\mathcal{C}} z^{-j-1} \frac{\left(q^{-b+d} z ; q\right)_{\infty}\left(q^{b-d+1} / z ; q\right)_{\infty}}{\left(q^{d} z ; q\right)_{\infty}\left(q^{c+2-d} / z ; q\right)_{\infty}} d z, \quad j=0, \pm 1, \pm 2, \ldots$

Thus, the moment functional in Theorem 3.1 satisfies

$$
\mathcal{M}^{(b, c, d)}\left[z^{-j}\right]=\frac{\tau^{(b, c)}}{2 \pi i} \int_{\mathcal{C}} z^{-j-1} \frac{\left(q^{-b+d} z ; q\right)_{\infty}\left(q^{b-d+1} / z ; q\right)_{\infty}}{\left(q^{d} z ; q\right)_{\infty}\left(q^{c+2-d} / z ; q\right)_{\infty}} d z
$$

for $j=0, \pm 1, \pm 2, \ldots$, which completes the proof of the theorem.

As a particular case, letting $b=0$ and $c+2 \neq 0,-1,-2, \ldots$ we have $\beta_{1}^{(0, c, d)}=$ $\frac{1-q^{c+1}}{1-q} q^{-d+1}$ and

$$
\beta_{n+1}^{(0, c, d)}=\alpha_{n+1}^{(0, c, d)}=\frac{1-q^{c+n+1}}{1-q^{n+1}} q^{-d+1}, \quad n \geq 1 .
$$

Moreover, $\mu_{0}^{(0, c, d)}=1$,

$$
\mu_{j}^{(0, c, d)}=0 \quad \text { and } \quad \mu_{-j}^{(0, c, d)}=\frac{\left(q^{-c-1} ; q\right)_{j}}{(q ; q)_{j}} q^{j(c+2-d)}, \quad j=1,2, \ldots .
$$

Furthermore, the following corollary holds.

Corollary 3.5.1. If $\mathcal{R} e(c+2)>\mathcal{R} e(d)>0$, then the sequence of polynomials $\left\{\mathcal{Q}_{n}^{(0, c, d)}\right\}$ given by

$$
\mathcal{Q}_{n}^{(0, c, d)}(z)=\frac{\left(q^{c+1} ; q\right)_{n}}{(q ; q)_{n}} q^{n(-d+1)}{ }_{2} \Phi_{1}\left(q^{-n}, q ; q^{-c-n} ; q, q^{-c+d-1} z\right), \quad n \geq 1
$$

apart from satisfying the three-term recurrence relation

$$
\mathcal{Q}_{n+1}^{(0, c, d)}(z)=\left(z+\frac{1-q^{c+n+1}}{1-q^{n+1}} q^{-d+1}\right) \mathcal{Q}_{n}^{(0, c, d)}(z)-\frac{1-q^{c+n+1}}{1-q^{n+1}} q^{-d+1} z \mathcal{Q}_{n-1}^{(0, c, d)}(z)
$$

for $n \geq 1$, with $\mathcal{Q}_{0}^{(0, c, d)}(z)=1$ and $\mathcal{Q}_{1}^{(0, c, d)}(z)=z+\frac{1-q^{c+1}}{1-q} q^{-d+1}$, satisfies the orthogonality relations

$$
\frac{1}{2 \pi i} \int_{\mathcal{C}} z^{-s} \mathcal{Q}_{n}^{(0, c, d)}(z) \frac{\left(q^{-d+1} / z ; q\right)_{\infty}}{\left(q^{c+2-d} / z ; q\right)_{\infty}} \frac{1}{z} d z=\delta_{n, s}, \quad 0 \leq s \leq n
$$

Moreover, uniformly on compact subsets of $|z|<\min \left\{q^{-\mathcal{R} e(c-d+2)}, q^{-\mathcal{R} e(-d+1)}\right\}$,

$$
\lim _{n \rightarrow \infty} \mathcal{Q}_{n}^{*(0, c, d)}(z)=\frac{\left(q^{\bar{c}-\bar{d}+2} z ; q\right)_{\infty}}{\left(q^{-\bar{d}+1} z ; q\right)_{\infty}} .
$$

As another particular case, letting $c=b$ and $b+1 \neq 0,-1,-2, \ldots$, we have

$$
\beta_{n}^{(b, b, d)}=\alpha_{n+1}^{(b, b, d)}=\frac{1-q^{n}}{1-q^{b+n}} q^{b-d+1}, \quad n \geq 1 .
$$

Moreover,

$$
\mu_{j}^{(b, b, d)}=\frac{\left(q^{-b} ; q\right)_{j}}{\left(q^{2} ; q\right)_{j}} q^{j d}, \quad j=0, \pm 1, \pm 2, \ldots .
$$

Furthermore, the following corollary can be stated. 
Corollary 3.5.2. If $b+1 \neq 0$ and $\mathcal{R} e(b+2)>\mathcal{R} e(d)>0$, then the sequence of polynomials $\left\{\mathcal{Q}_{n}^{(b, b, d)}\right\}$ given by

$$
\mathcal{Q}_{n}^{(b, b, d)}(z)=\frac{(q ; q)_{n}}{\left(q^{b+1} ; q\right)_{n}} q^{n(b-d+1)} \sum_{j=0}^{n} \frac{\left(q^{b+1} ; q\right)_{j}}{(q ; q)_{j}} q^{-j(b-d+1)} z^{j}, \quad n \geq 1,
$$

satisfies the orthogonality relations

$$
\frac{1}{2 \pi i} \frac{(1-q)}{\left(1-q^{b+1}\right)} \int_{\mathcal{C}} z^{-s} \mathcal{Q}_{n}^{(b, b, d)}(z) \frac{\left(q^{-b+d} z ; q\right)_{\infty}}{\left(q^{d} z ; q\right)_{\infty}} \frac{z-q^{b-d+1}}{z^{2}} d z=\delta_{n, s}, \quad 0 \leq s \leq n .
$$

Moreover, uniformly on compact subsets of $|z|<q^{-\mathcal{R} e(b-d+1)}$,

$$
\lim _{n \rightarrow \infty} \mathcal{Q}_{n}^{*(b, b, d)}(z)=\frac{1}{\left(1-q^{\bar{b}-\bar{d}+1} z\right)} .
$$

\section{4. $q$-SZEGŐ POLYNOMIALS}

From (3.8) the moment functional $\mathcal{M}^{(b, c, d)}$ is easily seen to be positive definite if $b \neq-1,-2, \ldots, c-b+1 \neq-1,-2, \ldots, \mathcal{R} e(c+2)>\mathcal{R} e(d)>0, \overline{-b+d}=b-d+1$ and $\bar{d}=c+2-d$. That is, with the restrictions

$$
c=b+\bar{b}-1, \quad d+\bar{d}=b+\bar{b}+1 \quad \text { and } \mathcal{R} e(b)>-1 / 2,
$$

the moment functional $\mathcal{M}^{(b, c, d)}$ is positive definite, and hence the polynomials $Q_{n}^{(b, c, d)}$ are the associated Szegö polynomials.

Hence, setting

$$
b=\lambda-i \eta, \quad c=2 \lambda-1 \quad \text { and } \quad d=\frac{1}{2}+\lambda+i \phi,
$$

if $\lambda>-1 / 2$, our special case of Ramanujan identity (3.7) becomes

$$
\sum_{-\infty}^{\infty} \frac{\left(q^{-\lambda+i \eta} ; q\right)_{n}}{\left(q^{\lambda+1+i \eta} ; q\right)_{n}} q^{n\left(\frac{1}{2}+\lambda+i \phi\right)} z^{n}=\tilde{\tau}^{(\lambda, \eta)} \frac{\left(q^{\frac{1}{2}+i(\eta+\phi)} z ; q\right)_{\infty}\left(q^{\frac{1}{2}-i(\eta+\phi)} / z ; q\right)_{\infty}}{\left(q^{\frac{1}{2}+\lambda+i \phi} z ; q\right)_{\infty}\left(q^{\frac{1}{2}+\lambda-i \phi} / z ; q\right)_{\infty}}
$$

which holds for $q^{\lambda+1 / 2}<|z|<q^{-\lambda-1 / 2}$, where

$$
\tilde{\tau}^{(\lambda, \eta)}=\frac{(q ; q)_{\infty}\left(q^{2 \lambda+1} ; q\right)_{\infty}}{\left(q^{1+\lambda+i \eta} ; q\right)_{\infty}\left(q^{1+\lambda-i \eta} ; q\right)_{\infty}} .
$$

This means that we can write

$$
\begin{aligned}
\mathcal{M}^{(\lambda-i \eta, 2 \lambda-1, \lambda+i \phi+1 / 2)}\left[z^{-j}\right] & =\frac{\left(q^{-\lambda+i \eta} ; q\right)_{j}}{\left(q^{\lambda+1+i \eta} ; q\right)_{j}} q^{j\left(\frac{1}{2}+\lambda+i \phi\right)} \\
& =\int_{\mathcal{C}} z^{-j} d \mu^{(\lambda, \eta, \phi)}(z)=\int_{0}^{2 \pi} e^{-i j \theta} \omega^{(\lambda, \eta, \phi)}(\theta) d \theta,
\end{aligned}
$$

for $j=0, \pm 1, \pm 2, \ldots$, where $\omega^{(\lambda, \eta, \phi)}(\theta) d \theta=d \mu^{(\lambda, \eta, \phi)}\left(e^{i \theta}\right)$, with

$$
\frac{d \mu^{(\lambda, \eta, \phi)}(z)}{d z}=\frac{\tilde{\tau}^{(\lambda, \eta)}}{2 \pi i} \frac{1}{z} \frac{\left(q^{\frac{1}{2}+i(\eta+\phi)} z ; q\right)_{\infty}\left(q^{\frac{1}{2}-i(\eta+\phi)} / z ; q\right)_{\infty}}{\left(q^{\frac{1}{2}+\lambda+i \phi} z ; q\right)_{\infty}\left(q^{\frac{1}{2}+\lambda-i \phi} / z ; q\right)_{\infty}}
$$

and

$$
\omega^{(\lambda, \eta, \phi)}(\theta)=\frac{\tilde{\tau}^{(\lambda, \eta)}}{2 \pi} \frac{\left(q^{\frac{1}{2}+i(\eta+\phi)} e^{i \theta} ; q\right)_{\infty}\left(q^{\frac{1}{2}-i(\eta+\phi)} e^{-i \theta} ; q\right)_{\infty}}{\left(q^{\frac{1}{2}+\lambda+i \phi} e^{i \theta} ; q\right)_{\infty}\left(q^{\frac{1}{2}+\lambda-i \phi} e^{-i \theta} ; q\right)_{\infty}} .
$$

As expected, $\omega^{(\lambda, \eta, \phi)}(\theta)$ is a positive weight function in $[0,2 \pi]$. 
Adopting the notation $S_{n}^{(\lambda, \eta, \phi)}(z)=Q_{n}^{\left(\lambda-i \eta, 2 \lambda-1, \frac{1}{2}+\lambda+i \phi\right)}(z)$ we can write the following:

$$
\begin{aligned}
S_{n+1}^{(\lambda, \eta, \phi)}(z)=( & \left.z+\frac{1-q^{\lambda+i \eta+n}}{1-q^{\lambda-i \eta+n+1}} q^{\frac{1}{2}-i(\eta+\phi)}\right) S_{n}^{(\lambda, \eta, \phi)}(z) \\
& \quad-\frac{\left(1-q^{n}\right)\left(1-q^{2 \lambda+n}\right)}{\left(1-q^{\lambda-i \eta+n}\right)\left(1-q^{\lambda-i \eta+n+1}\right)} q^{\frac{1}{2}-i(\eta+\phi)} z S_{n-1}^{(\lambda, \eta, \phi)}(z), \quad n \geq 1,
\end{aligned}
$$

with $S_{0}^{(\lambda, \eta, \phi)}(z)=1$ and $S_{1}^{(\lambda, \eta, \phi)}(z)=\left(z+\frac{1-q^{\lambda+i \eta}}{1-q^{\lambda-i \eta+1}} q^{\frac{1}{2}-i(\eta+\phi)}\right)$. Moreover,

$$
S_{n}^{(\lambda, \eta, \phi)}(0)=\frac{\left(q^{\lambda+i \eta} ; q\right)_{n}}{\left(q^{1+\lambda-i \eta} ; q\right)_{n}} q^{n\left[\frac{1}{2}-i(\eta+\phi)\right]}, \quad n \geq 1 .
$$

Hence, in particular, using Theorems 3.3 and 3.5 we have

Theorem 4.1. If $\lambda, \eta, \phi \in \mathbb{R}$ and $\lambda>-1 / 2$, then the polynomials

$$
S_{n}^{(\lambda, \eta, \phi)}(z)=\frac{\left(q^{\lambda+i \eta} ; q\right)_{n} q^{n\left[\frac{1}{2}-i(\eta+\phi)\right]}}{\left(q^{\lambda+1-i \eta} ; q\right)_{n}}{ }_{2} \Phi_{1}\left(q^{-n}, q^{\lambda+1-i \eta} ; q^{-\lambda-n+1-i \eta} ; q, q^{\frac{1}{2}-\lambda+i \phi} z\right)
$$

are the monic Szegö polynomials satisfying

$$
\int_{0}^{2 \pi} \overline{S_{n}^{(\lambda, \eta, \phi)}\left(e^{i \theta}\right)} S_{m}^{(\lambda, \eta, \phi)}\left(e^{i \theta}\right) \omega^{(\phi, \eta, \lambda)}(\theta) d \theta=\left[\kappa_{n}^{(\lambda, \eta)}\right]^{-2} \delta_{n, m}, \quad n, m=0,1,2, \ldots,
$$

with respect to the weight function $\omega^{(\lambda, \eta, \phi)}(\theta)$ given by (4.1). Here,

$$
\left[\kappa_{n}^{(\lambda, \eta)}\right]^{-2}=\rho_{n}^{(\lambda-i \eta, 2 \lambda-1)}=\frac{(q ; q)_{n}\left(q^{2 \lambda+1} ; q\right)_{n}}{\left(q^{\lambda+1+i \eta} ; q\right)_{n}\left(q^{\lambda+1-i \eta} ; q\right)_{n}} .
$$

Moreover, these polynomials satisfy the Szegö recurrence relation

$$
S_{n}^{*(\lambda, \eta, \phi)}(z)=\overline{a_{n}^{(\lambda, \eta, \phi)}} z S_{n-1}^{(\lambda, \eta, \phi)}(z)+S_{n-1}^{*(\lambda, \eta, \phi)}(z), \quad n \geq 1,
$$

where the reflection (or Verblunsky) coefficients $a_{n}^{(\lambda, \eta, \phi)}=S_{n}^{(\lambda, \eta, \phi)}(0)$ are given by (4.2).

Now using Theorem 3.4 we can state the following. Let $\lambda, \eta, \phi \in \mathbb{R}, \quad \lambda>-1 / 2$ and $\sigma=\min \left\{q^{-1 / 2}, q^{-\lambda-1 / 2}\right\}$. Then uniformly on compact subsets of $|z|<\sigma$,

$$
\lim _{n \rightarrow \infty} S_{n}^{*(\lambda, \eta, \phi)}(z)=\frac{\left(q^{\lambda+\frac{1}{2}+i \phi} z ; q\right)_{\infty}}{\left(q^{\frac{1}{2}+i(\eta+\phi)} z ; q\right)_{\infty}} .
$$

Moreover,

$$
\sum_{n=1}^{\infty}\left|a_{n}^{(\lambda, \eta, \phi)}\right|^{2}=\left|1-q^{\lambda+i \eta}\right|^{2} \sum_{n=1}^{\infty} \frac{q^{n}}{\left|1-q^{n+\lambda+i \eta}\right|^{2}} \leq\left|1-q^{\lambda+i \eta}\right|^{2} \sum_{n=1}^{\infty} \frac{q^{n}}{\left(1-q^{n+\lambda}\right)^{2}}<\infty .
$$

This last result means that the Szegó condition

$$
\frac{1}{2 \pi} \int_{0}^{2 \pi} \log \left(\omega^{(\lambda, \eta, \phi)}(\theta)\right) d \theta>-\infty
$$

holds and we can now give an expression for the associated Szegö function

$$
D^{(\lambda, \eta, \phi)}(z)=\exp \left(\frac{1}{4 \pi} \int_{0}^{2 \pi} \frac{e^{i \theta}+z}{e^{i \theta}-z} \log \left(\omega^{(\lambda, \eta, \phi)}(\theta)\right) d \theta\right) .
$$


Theorem 4.2. If $\lambda, \eta, \phi \in \mathbb{R}$ and $\lambda>-1 / 2$, then for $|z|<1$,

$$
D^{(\lambda, \eta, \phi)}(z)=\sqrt{\frac{\Gamma_{q}(2 \lambda+1)}{\Gamma_{q}(\lambda+1-i \eta) \Gamma_{q}(\lambda+1+i \eta)}} \frac{\left(q^{\frac{1}{2}+i(\eta+\phi)} z ; q\right)_{\infty}}{\left(q^{\lambda+\frac{1}{2}+i \phi} z ; q\right)_{\infty}} .
$$

Proof. Since, $\kappa_{n}^{(\lambda, \eta)} S_{n}^{*(\lambda, \eta, \phi)} \rightarrow\left[D^{(\lambda, \eta, \phi)}(z)\right]^{-1}$ for $|z|<1$ (see [23, p. 144]), the result follows from part $c$ ) of Theorem 3.2 and from (4.3).

\section{ACKNOWLEDGEMENTS}

The authors would like to thank Professor Zhedanov for valuable bibliographic information. The authors would also like to thank Professor Ismail for pointing out that the orthogonality relation in Theorem 4.1 is a special case of a biorthogonality relation involving polynomials attributed in his book [13] to Pastro [21].

\section{REFERENCES}

[1] E.X.L. de Andrade, C.F. Bracciali and A. Sri Ranga, Another connection between orthogonal polynomials and $L$-orthogonal polynomials, J. Math. Anal. Appl., 330 (2007), 114-132. MR2298161 (2008f:42026)

[2] G.E. Andrews, R. Askey and R. Roy, "Special Functions", Encyclopedia of Mathematics and its Applications, Cambridge University Press, 2000. MR.1688958 (2000g:33001)

[3] R. Askey, Ramanujan's extensions of the gamma and beta functions, Amer. Math. Monthly 87 (1980), 346-359. MR567718 (82g:01030)

[4] R. Askey (editor), "Gábor Szegő: Collected Papers. Volume 1", Contemporary Mathematicians, Birkhäuser, Boston, 1982. MR674482 (84d:01082a)

[5] A. Cachafeiro, F. Marcellán and C. Prez, Orthogonal polynomials with respect to the sum of an arbitrary measure and a Bernstein-Szegö measure, Adv. Comput. Math., 26 (2007), 81-104. MR2350346 (2008m:33032)

[6] R. Cruz-Barroso, P. González-Vera and F. Perdomo-Pío, Quadrature formulas associated with Rogers-Szegő polynomials, Comput. Math. Appl., 57 (2009), 308-323. MR 2488385 (2009k:65040)

[7] L. Daruis, O. Njåstad, and W. Van Assche, Szegő quadrature and frequency analysis, Electron. Trans. Numer. Anal., 19 (2005), 48-57. MR2149269 (2006e:41057)

[8] G. Gasper and M. Rahman, "Basic Hypergeometric Series", Cambridge Univ. Press, Cambridge, 1990. MR1052153(91d:33034)

[9] Ya.L. Geronimus, "Orthogonal Polynomials", Amer. Math. Soc. Transl., Ser. 2, vol. 108, American Mathematical Society, Providence, RI, 1977.

[10] L. Golinskii and A. Zlatoš, Coefficients of orthogonal polynomials on the unit circle and higher-order Szegő theorems, Constr. Approx., 26 (2007), 361-382. MR2335688(2008k:42080)

[11] E. Hendriksen and H. van Rossum, Orthogonal Laurent polynomials, Indag. Math. (Ser. A), 48 (1986), 17-36. MR834317 (87j:30008)

[12] M.E.H. Ismail, A simple proof of Ramanujan's ${ }_{1} \Psi_{1}$ sum, Proc. Amer. Math. Soc., 63 (1977), 185-186. MR0508183 (58:22695)

[13] M.E.H. Ismail, "Classical and Quantum Orthogonal Polynomials in One Variable", Cambridge Univ. Press, Cambridge, 2005. MR2191786 (2007f:33001)

[14] W.B. Jones and W.J. Thron, "Continued Fractions. Analytic Theory and Applications", Encyclopedia of Mathematics and its Applications, vol. 11, Addison-Wesley, Reading, MA, 1980. MR.595864 (82c:30001)

[15] W.B. Jones and W.J. Thron, Survey of continued fraction methods of solving moment problems, in: Analytic Theory of Continued Fractions, Lecture Notes in Math. 932, Springer, Berlin, 1981. MR690450 (84b:30002)

[16] R. Koekoek and R. Swarttouw, "The Askey-scheme of hypergeometric orthogonal polynomials and its q-analogue", Reports of the Faculty of Technical Mathematics and Informatics 98-17, Delft University of Technology, Delft, 1998.

[17] R.L. Lamblém, J.H. McCabe, M.A. Piñar and A. Sri Ranga, Szegő type polynomials and para-orthogonal polynomials, J. Math. Anal. Appl., 370 (2010), 30-41. MR2651127 
[18] L. Lorentzen and H. Waadeland, "Continued Fractions with Applications", Studies in Computational Mathematics, vol. 3, North-Holland, Amsterdam, 1992. MR.1172520 (93g:30007)

[19] A.L. Lukashov and F. Peherstorfer, Zeros of polynomials orthogonal on two arcs of the unit circle, J. Approx. Theory, 132 (2005), 42-71. MR2110575 (2006g:42045)

[20] A. Martínez-Finkelshtein, K.T.-R. McLaughlin and E.B. Saff, Szegő orthogonal polynomials with respect to an analytic weight: Canonical representation and strong asymptotics, Constr. Approx., 24 (2006), 319-363. MR2253965 (2007e:42029)

[21] P.I. Pastro, Orthogonal polynomials and some $q$-beta integrals of Ramanujan, J. Math. Anal. Appl., 112 (1985), 517-540. MR813618(87c:33015)

[22] J. Petronilho, Orthogonal polynomials on the unit circle via a polynomial mapping on the real line, J. Comput. Appl. Math., 216 (2008), 98-127. MR2421843 (2009e:42054)

[23] B. Simon, "Orthogonal Polynomials on the Unit Circle. Part 1. Classical Theory", American Mathematical Society Colloquium Publications, vol. 54, part 1, American Mathematical Society, Providence, RI, 2004. MR2105088(2006a:42002a)

[24] B. Simon, "Orthogonal Polynomials on the Unit Circle. Part 2. Spectral Theory", American Mathematical Society Colloquium Publications, vol. 54, part 2, American Mathematical Society, Providence, RI, 2004. MR2105089 (2006a:42002b)

[25] B. Simon, Equilibrium measures and capacities in spectral theory, Inverse Probl. Imaging, 1 (2007), 713-772. MR2350223 (2008k:31003)

[26] L.J. Slater, "Generalized Hypergeometric Functions", Cambridge Univ. Press, Cambridge, 1966. MR0201688 (34:1570)

[27] A. Sri Ranga, Szegő polynomials from hypergeometric functions, Proc. Amer. Math. Soc., 138 (2010), 4259-4270. MR2680052

[28] G. Szegő, Über Beiträge zur theorie der toeplitzschen formen, Math. Z., 6 (1920), 167-202. MR.1544404

[29] G. Szegö, Über Beiträge zur theorie der toeplitzschen formen, II, Math. Z., 9 (1921), 167-190. MR 1544462

[30] G. Szego", "Orthogonal Polynomials", 4th ed., Amer. Math. Soc. Colloq. Publ., vol. 23, Amer. Math. Soc., Providence, RI, 1975. MR0372517 (51:8724)

[31] L. Vinet and A. Zhedanov, Spectral transformations of the Laurent biorthogonal polynomials, II. Pastro polynomials, Canad. Math. Bull., 44 (2001), 337-345. MR1847496 (2002g:33022)

[32] A. Zhedanov, The "classical" Laurent biorthogonal polynomials, J. Comput. Appl. Math., 98 (1998), 121-147. MR1656982(99k:33065)

Pós-Graduação em Matemática, ibilce, UnesP-Universidade Estadual Paulista, 15054-000, SÃo José do Rio Preto, SP, Brazil

E-mail address: isacosta.mat@bol.com.br

Departamento de Matemática Aplicada II, E.T.S.I. Industriales, Universidade de Vigo, Campus Lagoas-Marcosende, 36310 Vigo, Spain

E-mail address: egodoy@dma.uvigo.es

Pós-Graduação em Matemática, ibilce, UneSP-Universidade Estadual Paulista, 15054-000, SÃo José do Rio Preto, SP, BraziL

E-mail address: parareginae@hotmail.com

Departamento de Ciências de ComputaÇão e Estatística, ibilce, UneSP-Universidade Estadual Paulista, 15054-000, São José do Rio Preto, SP, Brazil

E-mail address: ranga@ibilce.unesp.br 